hep-th/0512249

TU-762

HD-THEP-06-4

\title{
Gravitational Kaluza-Klein Modes in Warped Superstring Compactification
}

\author{
Tatsuya Noguchi * \\ Institute for Theoretical Physics, Heidelberg University, \\ D-69120 Heidelberg, Germany \\ Masahiro Yamaguchi ${ }^{\dagger}$ and Masakazu Yamashita ${ }^{\ddagger}$ \\ Department of Physics, Tohoku University, \\ Sendai 980-8578, Japan
}

\begin{abstract}
The Kaluza-Klein (KK) modes of graviton are studied in the IIB superstring compactification where the warped geometry is realized at the Klebanov-Strassler (KS) throat. Knowledge of the metric of the KS throat enables us to determine their wave functions with good accuracy, without any further specification of the rest of CalabiYau space, owing to the localization of the KK modes. Mass spectrum and couplings to the four dimensional fields are computed for some type of the KK modes, and compared to those of the well-known Randall-Sundrum model. We find that the properties of the KK modes of the two models are very different in both the masses and the couplings, and thus they are distinguishable from each other experimentally.
\end{abstract}

\footnotetext{
*noguchi@tphys.uni-heidelberg.de

†yama@tuhep.phys.tohoku.ac.jp

¥ yamasita@tuhep.phys.tohoku.ac.jp
} 


\section{Introduction}

The warped extra dimension advocated by Randall and Sundrum (RS) [1, 2] is a fascinating idea to solve the naturalness problem associated with the scale of electro-weak symmetry breaking. In this model, the spacetime is a slice of 5-dimensional Anti-de Sitter spacetime with two boundaries of four dimensions. The standard model (SM) is assumed to be confined on one of the boundaries, called the infra-red (IR) brane. The characteristic scale on the IR brane is re-normalized down to the $\mathrm{TeV}$ scale with an appropriate choice of the warp factor, providing a solution to the naturalness problem. Besides, the Kaluza-Klein (KK) modes of graviton are localized near the IR brane, and hence their masses and couplings to the four dimensional fields are characterized by the $\mathrm{TeV}$ scale, with interesting phenomenological consequences.

Despite its attractive features, the RS model as it is has some drawbacks. One point is that the IR brane on which the SM fields are confined has a negative tension, which makes its physical interpretation difficult. Another point is that it has a sharp cut at the IR brane, which is somewhat ad hoc. Related to this, the warp factor is given by hand, not fixed by some dynamical mechanism (see however Ref. [3]). All these arguments call for better understanding of the foundation of this fascinating idea. In string theories, the warped metric is natural in the presence of D-branes. In fact the AdS/CFT [4] correspondence makes one anticipate stringy realization of the warped extra dimension [5, 6]. An explicit realization has been given in Ref. [7. The basic ingredient is type IIB superstring theory compactified on a Calabi-Yau manifold with the Klebanov-Strassler (KS) throat, i.e. a deformed conifold with non-vanishing RR and NS fluxes present. The KS throat has asymptotically AdS metric, and hence the warped geometry is obtained at the KS throat. The complex moduli are fixed by the fluxes, so is the warp factor. The latter determines the characteristic scale of the standard model, provided that the SM sector resides on D-branes put additionally at the apex of the KS throat.

The purpose of this paper is to investigate some properties of the warped string compactification described above. In particular, we will look into the Kaluza-Klein modes of the graviton in this case. As in the case of the RS model, the wave functions of the KK modes will be localized on the infra-red side, around the apex of the KS throat. This allows us to compute the masses and wave functions of the KK modes in good approximation, without the information of the complicated metric of the attached Calabi-Yau manifold.

Though the KS geometry has asymptotically AdS metric, its structure in the far IR side is quite different. Thus one can expect that the profiles of the KK modes also differ from those of the RS model. As we will show later, this is indeed the case. We compute the KK modes which depend only on the radial direction of the deformed conifold and compare them with 
those of the RS model. We find that the mass eigenvalues are very different from each other. Moreover, we compute the couplings of the KK modes to the four dimensional fields on the IR brane, which are inferred from the values of the wave functions there. It is known that in the RS model all KK modes couple to the SM particles with the same strength. We find it no longer the case in this string setup.

In addition to the radial modes which we will study in this paper, there are modes which depend also on angular directions. The angular dependence of the KS geometry is somewhat involved, which makes difficult the study of such modes. We shall make a brief comment on it, leaving the detailed study of the angular modes for future investigation.

The organization of the paper is as follows. In the next section, we study the KK modes of the warped string compactification with the KS throat. We will solve the wave equations for the KK modes numerically, and obtain their mass eigenvalues and their couplings to the four dimensional fields, read from a value of the wave function at the IR end, for the first several states. In section 3, after reviewing the properties of the KK modes of the RS model, we make a comparison of the two models. A brief comment on the angular dependent modes is made in section 4 . The final section is devoted to conclusions.

\section{KK spectrum and couplings in warped superstring compactification}

We consider the warped compactification in superstring theories, realized on the basis of a deformed conifold [7]. The background metric of the deformed conifold in the presence of fluxes for type IIB supergravity was solved by Klebanov and Strassler (the KS geometry) [8]. In the region far from the top of the deformed conifold, the metric approaches the AdS one, and thus one can use the KS geometry as a part of the warped compactification. Since the deformed conifold is non-compact with its UV end open, one has to glue it to a Calabi-Yau manifold to make the extra space dimensions compact, which eventually reproduce a finite four dimensional gravity.

Here we study the gravitational KK modes in this setup. It is known that wave functions are localized near the apex of the warped throat like in the case of the RS model, and thus remain unaffected by the UV structure, i.e. the CY manifold glued into the deformed conifold. Therefore, we perform the calculation of the gravitational KK modes simply on the KS geometry itself, without any further compactification. 


\subsection{KS solution in the Type IIB supergravity}

We begin by briefly reviewing the type IIB supergravity solution on a deformed conifold which we call the KS solution [8].

The deformed conifold is a 3 -dimensional complex space defined by the equation in $\mathbb{C}^{4}$,

$$
\left(z^{1}\right)^{2}+\left(z^{2}\right)^{2}+\left(z^{3}\right)^{2}+\left(z^{4}\right)^{2}=\epsilon^{2}
$$

This manifold is a cone, whose base space is topologically equivalent to $\mathbf{S}^{3} \times \mathbf{S}^{2}$ [9]. In contrast to the singular conifold $(\epsilon=0)$, the base space of the deformed conifold does not shrink to a point even at $\tau=0$ : it turns out the (squashed) $\mathbf{S}^{3}$. We would like to note that this non-compact manifold should be regarded as a local description of some Calabi-Yau manifold, where the large $\tau$ region of the conifold is glued to a compact CY manifold.

In Ref. 8], a non-singular supergravity solution of Type IIB superstring theory compactified on the deformed conifold was given, with $M$ fractional D3-branes at the apex:

$$
M=\int_{\mathbf{S}^{3}} \widetilde{F}_{(3)} .
$$

The fractional D3-branes, defined as D5-branes wrapped around the collapsed 2-cycles $\mathbf{S}^{2}$, serve as sources for the magnetic RR 3-form flux over $S^{3}$, and make the 2 -form field $B_{(2)}$ dependent on the radial coordinate.

The solution of the type IIB supergravity on the deformed conifold is given as follows under the assumption that the fractional 3-branes are smeared over the $S^{3}$ at $\tau=0$,

$$
\begin{aligned}
d s_{(10)}^{2}= & h^{-1 / 2}(\tau) \eta_{\mu \nu} d x^{\mu} d x^{\nu}+h^{1 / 2}(\tau) d s_{\text {deformed }}^{2} \\
d s_{\text {deformed }}^{2}= & \frac{1}{2} \epsilon^{4 / 3} K(\tau) \times\left[\frac{1}{3 K^{3}(\tau)}\left[d \tau^{2}+\left(g^{5}\right)^{2}\right]+\cosh ^{2}\left(\frac{\tau}{2}\right)\left[\left(g^{3}\right)^{2}+\left(g^{4}\right)^{2}\right]\right. \\
& \left.+\sinh ^{2}\left(\frac{\tau}{2}\right)\left[\left(g^{1}\right)^{2}+\left(g^{2}\right)^{2}\right]\right]
\end{aligned}
$$

where

$$
\begin{aligned}
K(\tau) & =\frac{(\sinh (2 \tau)-2 \tau)^{1 / 3}}{2^{1 / 3} \sinh \tau} \\
h(\tau) & =2^{2 / 3} \epsilon^{-8 / 3}\left(g_{\mathrm{st}} M \alpha^{\prime}\right)^{2} I(\tau) \\
I(\tau) & =\int_{\tau}^{\infty} d x \frac{x \operatorname{coth} x-1}{\sinh ^{2} x}(\sinh 2 x-2 x)^{1 / 3}
\end{aligned}
$$

Note that in the asymptotic region with a large $\tau$ this metric becomes the AdS one:

$$
d s_{(10)}^{2} \sim \frac{R^{2}}{U^{2}} d s_{(4)}^{2}+\frac{U^{2}}{R^{2}}\left[d U^{2}+U^{2} d s_{\mathbb{T}^{1,1}}^{2}\right]
$$


where

$$
U^{3}=\left(\frac{3}{2}\right)^{3 / 2} \epsilon^{2} \cosh \tau .
$$

In terms of coordinate 1 -forms $g^{i}(i=1, \cdots 5)$ dependent on the coordinates of the base space $\left\{\theta^{1,2}, \phi^{1,2}, \psi\right\}$, the 3 -form fluxes and the 5 -form fluxes satisfying the equation of motion are formulated as follows

$$
\begin{aligned}
& g_{\mathrm{st}} \widetilde{F}_{(3)}=\frac{g_{\mathrm{st}} M \alpha^{\prime}}{2}\left[g^{5} \wedge g^{3} \wedge g^{4}(1-F(\tau))+g^{5} \wedge g^{1} \wedge g^{2} F(\tau)\right. \\
&\left.+F^{\prime}(\tau) d \tau \wedge\left(g^{1} \wedge g^{3}+g^{2} \wedge g^{4}\right)\right], \\
& H_{(3)}= \frac{g_{\mathrm{st}} M \alpha^{\prime}}{2}\left[d \tau \wedge\left(f^{\prime}(\tau) g^{1} \wedge g^{2}+k^{\prime}(\tau) g^{3} \wedge g^{4}\right)\right. \\
&\left.\quad+\frac{1}{2}(k(\tau)-f(\tau)) g^{5} \wedge\left(g^{1} \wedge g^{3}+g^{2} \wedge g^{4}\right)\right], \\
& g_{\mathrm{st}} \widetilde{F}_{(5)}= B_{(2)} \wedge \widetilde{F}_{(3)} \\
&= \frac{\left(g_{\mathrm{st}} M \alpha^{\prime}\right)^{2}}{4} l(\tau) g^{1} \wedge g^{2} \wedge g^{3} \wedge g^{4} \wedge g^{5},
\end{aligned}
$$

where

$$
\begin{aligned}
F(\tau) & =\frac{\sinh \tau-\tau}{2 \sinh \tau}, \\
f(\tau) & =\frac{\tau \operatorname{coth} \tau-1}{2 \sinh \tau}(\cosh \tau-1), \\
k(\tau) & =\frac{\tau \operatorname{coth} \tau-1}{2 \sinh \tau}(\cosh \tau+1), \\
l(\tau) & =\frac{\tau \operatorname{coth} \tau-1}{4 \sinh ^{2} \tau}(\sinh 2 \tau-2 \tau) .
\end{aligned}
$$

The KS solution should be interpreted as a natural embedding of warped geometry in string theory. The warp factor at the top of the deformed conifold is computed in Ref. [7], with the characteristic mass scale

$$
m_{\text {scalar }} \sim M_{\text {Planck }} \exp \left(-\frac{2 \pi K}{3 M g_{\mathrm{st}}}\right),
$$

where $M, K$ are RR and NS fluxes respectively.

\section{$2.2 \quad$ Numerical results of KK modes}

We consider the gravitational KK modes in the deformed conifold. Here we focus on the KK modes whose wave functions depend only on radial direction of the deformed conifold. This 
is partly because these modes would correspond to the KK modes in the RS model, which has a single extra space dimension.

The Einstein's equation of the type IIB supergravity theory is known as

$$
\begin{aligned}
\mathcal{R}_{M N}= & -\frac{1}{8} g_{M N}\left(\left|H_{(3)}\right|^{2}+g_{\mathrm{st}}^{2}\left|\widetilde{F}_{(3)}\right|^{2}\right) \\
& +\frac{1}{2}\left(\frac{1}{2 !} H_{(3) M R_{1} R_{2}} H_{(3) N} R_{1} R_{2}+\frac{g_{\mathrm{st}}^{2}}{2 !} \widetilde{F}_{(3) M R_{1} R_{2}} \widetilde{F}_{(3) N} R_{1} R_{2}\right. \\
& \left.+\frac{g_{\mathrm{st}}^{2}}{2 \cdot 4 !} \widetilde{F}_{(5) M R_{1} R_{2} R_{3} R_{4}} \widetilde{F}_{(5) N} R_{1} R_{2} R_{3} R_{4}\right),
\end{aligned}
$$

where and hereafter we define that Greek indices run over 4D Minkowski space, Roman ones over the internal manifold, and capital Roman ones over 10D spacetime.

The linearized equation with respect to the metric fluctuation $h_{\mu \nu}(x, \tau)$ turns out to be

$$
\begin{aligned}
-\frac{1}{2} \nabla^{R} \nabla_{R} h_{\mu \nu}(x, \tau)+\frac{1}{2} \nabla^{R} \nabla_{\mu} h_{R \nu}(x, \tau)+\frac{1}{2} \nabla^{R} \nabla_{\nu} h_{R \mu}(x, \tau) \\
=-\frac{1}{8} h_{\mu \nu}(x, \tau)\left(\left|H_{(3)}\right|^{2}+g_{\mathrm{st}}^{2}\left|\widetilde{F}_{(3)}\right|^{2}\right)+\frac{g_{\mathrm{st}}^{2}}{4}\left|\star \mathcal{F}_{(5)}\right|^{2} h_{\mu \nu}(x, \tau),
\end{aligned}
$$

where $h_{\mu \nu}(x, \tau)$ is defined as

$$
g_{\mu \nu}=h^{-1 / 2}(\tau) \eta_{\mu \nu}+h_{\mu \nu}(x, \tau)
$$

Assuming that the fluctuation of metric in the axial gauge would be decomposed into the $\tau$-dependent part and a plane wave solution with a definite momentum $k$ as,

$$
h_{\alpha \beta}\left(x^{\mu}, \tau\right)=\psi(\tau) e^{i k \cdot x} \quad(\alpha, \beta=1,2,3),
$$

we find that Eq. (44) turns out

$$
\psi^{\prime \prime}+A(\tau) \psi^{\prime}+B(\tau) \psi=-\frac{2^{2 / 3}\left(g_{\mathrm{st}} M \alpha^{\prime}\right)^{2}}{\epsilon^{4 / 3}} I(\tau) G_{55}(\tau) k^{2} \psi
$$

where

$$
A(\tau)=\frac{\partial \ln G_{99}(\tau)}{\partial \tau}+\frac{\partial \ln G_{77}(\tau)}{\partial \tau}+\frac{\partial \ln I(\tau)}{\partial \tau}
$$

and

$$
\begin{aligned}
B(\tau)= & -\frac{1}{4} \cdot \frac{2^{1 / 3}}{8 I(\tau) G_{77}^{2}(\tau)}\left[(1-F(\tau))^{2}+{k^{\prime}}^{2}(\tau)\right] \\
& -\frac{1}{4} \cdot \frac{2^{1 / 3}}{16 G_{77}(\tau) G_{99}(\tau)}\left[(k(\tau)-f(\tau))^{2}+4{F^{\prime}}^{2}(\tau)\right] \\
& -\frac{1}{4} \cdot \frac{2^{1 / 3}}{8 I(\tau) G_{99}^{2}(\tau)}\left(f^{\prime 2}(\tau)+F^{2}(\tau)\right)+\frac{1}{4 I(\tau)^{2}} I^{\prime 2}(\tau) \\
& -\frac{2^{5 / 3} l^{2}(\tau)}{I^{2}(\tau) K^{4}(\tau)\left(\cosh ^{2} \tau-1\right)^{2}} .
\end{aligned}
$$


We adopt here the notation in Ref. [12], where $G_{i i}(i=5, \cdots, 10)$ take the following forms, defined from the KS metric :

$$
\begin{aligned}
& G_{55}(\tau)=G_{66}(\tau)=\frac{1}{6} K^{-2}(\tau), \\
& G_{77}(\tau)=G_{88}(\tau)=\frac{1}{2} K(\tau) \cosh ^{2}(\tau / 2), \\
& G_{99}(\tau)=G_{1010}(\tau)=\frac{1}{2} K(\tau) \sinh ^{2}(\tau / 2) .
\end{aligned}
$$

As for the boundary condition, we require that $d \psi / d \tau$ should be zero at one boundary $\tau=0$, and that $\psi$ should approach to $e^{-4 \tau / 3}$ in the large $\tau$ region. The latter requirement comes from the fact that the background metric approaches the AdS.

We obtained the mass spectrum for the graviton excitation in the KS model through the shooting method [11, 12. In Table 1, we show the mass ratios to the first excited state and the difference between two neighboring ratios (left), and the coupling constants between KK modes and the boundary fields (right). The first KK mode has a mass $4.44 M_{\text {apex }}$, expressed in the unit

$$
M_{\text {apex }} \equiv \epsilon^{2 / 3} /\left(g_{\mathrm{st}} M \alpha^{\prime}\right)
$$

The ratios of the coupling constants to the first excited state are shown, read from the values of the normalized wave functions at the apex of the warped throat,

$$
g_{n} \propto \psi(\tau=0)
$$

\begin{tabular}{|c|c|c|}
\hline Level & Mass ratio & Difference \\
\hline 6th & 2.48 & 0.31 \\
5th & 2.17 & 0.31 \\
4th & 1.86 & 0.31 \\
3rd & 1.55 & 0.28 \\
2nd & 1.27 & 0.27 \\
1st & 1.00 & \\
\hline
\end{tabular}

\begin{tabular}{|c|c|}
\hline Level & Coupling ratio \\
\hline 6th & 0.80 \\
5 th & 0.89 \\
4th & 0.98 \\
3rd & 1.10 \\
2nd & 1.18 \\
1 st & 1.00 \\
\hline
\end{tabular}

Table 1: The mass ratios to the first excited state and the difference between them (left), and the coupling constants of the KK modes to the SM fields localized at the apex of the warped throat, normalized by the coupling of the first excited state (right) for the KS geometry. They are obtained through the shooting method, where we evaluated the boundary condition at a point sufficiently near to the apex, not exactly at the apex, for avoiding computational difficulty. 


\section{Comparison with Randall-Sundrum Model}

In this section, we would like to compare the results obtained for the warped string compactification with those of the Randall-Sundrum model.

In this model, the spacetime has five dimensions with one extra dimension compactified on $\mathbf{S}^{1} / \mathbb{Z}_{2}$. The Standard Model (SM) is thought to be localized on one of two fixed planes under $\mathbb{Z}_{2}$ transformation. Due to the (negative) cosmological constant $\Lambda(<0)$, the metric solution of 5D Einstein's equation is exponentially warped along 5 -th dimension $y$ :

$$
d s^{2}=e^{-2 k|y|} \eta_{\mu \nu} d x^{\mu} d x^{\nu}+d y^{2},
$$

where the curvature constant $k$ is related to $\Lambda$ as

$$
k^{2}=\frac{-\Lambda}{24 M^{3}},
$$

and $M$ is the fundamental mass scale. It is well known that this metric could be converted to the AdS one with some coordinate transformation. Two 3-branes with opposite tension are required to locate at each $\mathbb{Z}_{2}$ fixed point $y=0$ and $r_{c}$ (the radius of $\mathbf{S}^{1}$ ) so that the 5 th dimensional space could be static. These tensions $T_{ \pm}$are related to the cosmological constant through the curvature constant $k$ :

$$
\frac{T_{+}}{24 M^{3}}=k=-\frac{T_{-}}{24 M^{3}} .
$$

We assume that the SM fields reside on the negative tension 3-brane at $y=r_{\mathrm{c}}$ (called the IR brane), and obtain the characteristic scale, which is to be regarded as the mass scale of the Higgs boson

$$
m_{\text {Higgs }}=M e^{-k r_{c}},
$$

while the effective 4-dimensional Planck constant $M_{\mathrm{Pl}}$ is

$$
M_{\mathrm{Pl}}^{(4)}=\frac{M^{2}}{k}\left(1-e^{-k r_{c}}\right) \sim M .
$$

It follows from these relations that even with a moderate choice of $r_{\mathrm{c}}$ and $k$, one can reproduce an extremely small mass scale compared with $M$.

Next, we discuss the gravitational KK modes. We define the gravitational fluctuation $h_{\mu \nu}$ as in Ref. [2]

$$
g_{\mu \nu}=e^{-2 k|y|} \eta_{\mu \nu}+h_{\mu \nu} .
$$

The linearized Einstein's equation for $h_{\mu \nu}$ is described in the transverse and traceless gauge

$$
\left[-\frac{1}{2} \partial_{y}^{2}+2 k^{2}-2 k \delta(y)+2 k \delta\left(y-y_{c}\right)\right] \psi(y)=\frac{m^{2}}{2} e^{2 k|y|} \psi(y),
$$


where $\psi(y)$ is defined as $h_{\mu \nu}=\psi(y) e^{i k \cdot x}$. Note that the $\delta$-functions come from the positive and negative 3-branes. With the introduction of a new coordinate and a new wave function $z \equiv \operatorname{sgn}(y)\left(e^{k|y|}-1\right) / k$ and $\widehat{\psi} \equiv \psi e^{k|y| / 2}$, this equation becomes the Schrödinger type with a constant mass term,

$$
\left[-\frac{1}{2} \partial_{z}^{2}+\frac{15 k^{2}}{8(k|z|+1)^{2}}-\frac{3 k}{2} \delta(z)+\frac{3 k}{2\left(k z_{\mathrm{c}}+1\right)^{2}} \delta\left(z-z_{\mathrm{c}}\right)\right] \widehat{\psi}(z)=m^{2} \widehat{\psi}(z),
$$

and the metric becomes conformal

$$
d s^{2}=\frac{1}{(k|z|+1)^{2}}\left[\eta_{\mu \nu} d x^{\mu} d x^{\nu}+d z^{2}\right] .
$$

The boundary condition on $\widehat{\psi}(z)$ must be read from the condition that all the $\delta$-functions should disappear in Eq. (35) at $z=0_{+}$and $z=z_{c-}$, where $a_{ \pm}=a \pm \varepsilon$ with a infinitely small positive parameter $\varepsilon$,

$$
\begin{aligned}
{\left[\partial_{z} \widehat{\psi}(z)\right]_{z=0+} } & =\left[-\operatorname{sgn}(z) \frac{3 k}{2(k|z|+1)} \widehat{\psi}(z)\right]_{z=0+}, \\
{\left[\partial_{z} \widehat{\psi}(z)\right]_{z=z_{\mathrm{c}-}} } & =\left[+\operatorname{sgn}\left(z-z_{\mathrm{c}}\right) \frac{3 k}{2(k \zeta(z)+1)} \widehat{\psi}(z)\right]_{z=z_{\mathrm{c}-}}
\end{aligned}
$$

where $\zeta(z) \equiv-\left|z-z_{\mathrm{c}}\right|+z_{\mathrm{c}}$.

In Table 2, we show the mass spectrum for the KK-modes of the RS model with $k=1$ and $y_{\mathrm{c}}=6.64$, numerically computed through the shooting method [11, 12], where the masses lie at almost the same intervals. ${ }^{\S}$ The table also shows that the KK modes couple with the field localized at $z=z_{\mathrm{c}}$ with almost the same coupling constant, whose difference are of order $0.001 \%$. This universality is a characteristic feature of RS model [10]. The authors discussed this coupling constant universality analytically, under the assumption of $m_{n} / k \ll 1$, which is naturally satisfied when we set the value $k y_{c}$ for solving the hierarchy problem.

Our numerical observation of the coupling constant universality here for RS model would be important when we estimate the numerical error of the result in the previous section for the KS model, which might potentially accumulate in the far UV region: one could estimate it to be somehow similar to that of RS model, i.e., $0.001 \%$, because they have the same asymptotic behavior in the UV region.

We are ready to compare the KK spectrum of the warped string compactification with those of the RS model. Comparison of Table 1 with Table 2 shows the clear difference of the mass spectrum, for example, the ratios of the second KK mode to the first differ by 67 $\%$. In addition to the mass spectra, we would note that the difference can be seen on the

\footnotetext{
$\S^{\S}$ The IR cutoff $y_{c}=6.64$ is corresponding to that of the KS calculation in the previous section.
} 


\begin{tabular}{|c|c|c|}
\hline Level & Mass ratio & Difference \\
\hline 6th & 5.12 & 0.82 \\
5th & 4.30 & 0.82 \\
4th & 3.48 & 0.82 \\
3rd & 2.66 & 0.82 \\
2nd & 1.83 & 0.83 \\
1st & 1.00 & \\
\hline
\end{tabular}

\begin{tabular}{|c|c|}
\hline Level & Coupling ratio \\
\hline 6th & 1.00 \\
5 th & 1.00 \\
4 th & 1.00 \\
3 rd & 1.00 \\
2nd & 1.00 \\
1 st & 1.00 \\
\hline
\end{tabular}

Table 2: The mass ratios to the first excited state and the difference between them (left), and the coupling constants of the KK modes to the SM fields on the IR brane, normalized by the first excitation state (right).

coupling constants: the warped string compactification based on the KS geometry shows no longer the coupling constant universality observed in the RS model.

These differences will be a clear target of future collider experiments such as the Large Hadron Collider (LHC) and a future lepton collider [10, 13, where the difference of the masses and couplings leads to the difference in the loci and the widths of the s-channel resonances of the KK modes of the graviton.

At the end of this section, we would like to mention the difference between the warped string compactification and a slice of $\mathrm{AdS}_{5} \times \mathcal{M}$ [13]. In the latter case, on top of the KK modes which are identical to the RS model (a slice of $\mathrm{AdS}_{5}$ ), there are new KK modes whose wave functions depend on coordinates of $\mathcal{M}$. On the other hand, the KS geometry, which is used for the warped string compactification, cannot be decomposed this way. As we have seen, the modes with no angular dependence have different mass eigenvalues and wave functions from the RS model. Furthermore, angular dependent modes would have more complicated behavior than the case of the product of the $\mathrm{AdS}_{5}$ slice and $\mathcal{M}$, as we will discuss shortly. Thus, there appears clear difference between the two cases. It is interesting to investigate whether these two cases can clearly be distinguished experimentally, which is beyond the scope of the present paper.

\section{Angular Dependent Modes}

In this section, we make a brief comment on KK modes with angular dependence. As was discussed earlier, the deformed conifold is a cone whose base manifold is $\mathbf{S}^{3} \times \mathbf{S}^{2}$. At $\tau=0$ the metric degenerates into that of a round $\mathbf{S}^{3}$ :

$$
d s_{\text {deformed }}^{2} \sim \frac{1}{2} \epsilon^{4 / 3}\left(\frac{2}{3}\right)^{1 / 3}\left[\frac{1}{2}\left(g^{5}\right)^{2}+\left(g^{3}\right)^{2}+\left(g^{5}\right)^{2}\right],
$$


and $\mathbf{S}^{2}$ collapses there. The angular dependent part would be described by the spherical harmonics $Y_{l m}$ on $\mathbf{S}^{2}$ and the harmonics $Z_{p q r}$ on $\mathbf{S}^{3}$. The modes with $l=0$ do not vanish at the apex.

A slice of the KS geometry at $\tau \neq 0$ contains the round $\mathbf{S}^{2}$ and the squashed $\mathbf{S}^{3}$. Thus the modes with the vanishing angular momentum for the round $\mathbf{S}^{2}$ are characterized by the isometry of the squashed $\mathbf{S}^{3}$, i.e. $S U(2) \times U(1)$ [14]. Only these modes can couple to SM particles when the SM brane is at the top of the deformed conifold. On the other hand, if the SM brane is off the top of the deformed conifold $(\tau \neq 0)$, the modes having non-zero angular momentum associated to the $\mathbf{S}^{2}$ will have non-vanishing wave functions at the SM brane, and hence can couple to the SM particles. Interestingly the strength of the couplings will depend on the location of the brane off the top. We leave more detailed study on the angular dependent modes for future study.

\section{Conclusions and Discussion}

In this paper, we considered the KK modes of the graviton in the warped string compactification where the warped geometry is realized at the KS throat. Focusing on the modes with only radial direction dependence, we computed their masses and coupling constants to the SM particles which are thought to reside at the top of the deformed conifold. We compared these results with those of the RS model, and found clear difference between the two models, which suggests, interestingly, that that they are distinguishable from each other in future collider experiments.

Our analysis is based on Ref. [7, where complex moduli as well as dilaton are stabilized, while Kähler moduli remain unfixed. The issue of the stabilization of the latter moduli was addressed in Ref. [15]. In the KKLT setup, the Kähler moduli are stabilized by nonperturbative effects such as Euclidean D3-branes and gaugino condensation on D7-branes, and $\overline{\mathrm{D}}$-branes are put at the apex of the deformed conifold to lift up the otherwise negative vacuum energy. Here we would like to briefly discuss how they will affect the results we obtained. As we emphasized in this paper, the gravitational KK modes have wave functions localized near the apex of the deformed conifold. The non-perturbative configurations uplifting the Kähler moduli potential, as far as it is added in the UV Calabi-Yau region, does not affect substantially the KK mode wave functions. The existence of the $\overline{\mathrm{D}}$-branes opens a new possibility that the SM fields live on the $\overline{\mathrm{D}}$-branes. However as far as the low-energy behavior of the KK modes is concerned, whether the SM lives on the $\overline{\mathrm{D}}$-branes or on the D-branes makes no difference. We, thus, conclude that the inclusion of the KKLT setup does not change our results significantly. 
Much remains to be seen: firstly, we need to take into consideration also the modes with angular dependence along the deformed conifold, and secondly we should investigate experimental signatures of KK modes in more detail. We leave the study of these issues for future work.

\section{Acknowledgments}

We would like to thank H. Ishikawa for useful discussions. This work was supported in part by the Scientific Grants from the Ministry of Education, Science, Sports, and Culture of Japan, No. 16081202 and 17340062.

\section{Note Added}

After completion of this work, we received a preprint [16] which has some overlap with our work.

\section{References}

[1] L. Randall and R. Sundrum, Phys. Rev. Lett. 83, 3370 (1999) arXiv:hep-ph/9905221.

[2] L. Randall and R. Sundrum, Phys. Rev. Lett. 83, 4690 (1999) arXiv:hep-th/9906064.

[3] W. D. Goldberger and M. B. Wise, Phys. Rev. Lett. 83, 4922 (1999) arXiv:hep-ph/9907447.

[4] J. M. Maldacena, Adv. Theor. Math. Phys. 2, 231 (1998) [Int. J. Theor. Phys. 38, 1113 (1999)] arXiv:hep-th/9711200.

[5] H. L. Verlinde, Nucl. Phys. B 580, 264 (2000) arXiv:hep-th/9906182.

[6] C. S. Chan, P. L. Paul and H. L. Verlinde, Nucl. Phys. B 581, 156 (2000) arXiv:hep-th/0003236.

[7] S. B. Giddings, S. Kachru and J. Polchinski, Phys. Rev. D 66, 106006 (2002) arXiv:hep-th/0105097.

[8] I. R. Klebanov and M. J. Strassler, JHEP 0008, 052 (2000) arXiv:hep-th/0007191.

[9] P. Candelas and X. C. de la Ossa, Nucl. Phys. B 342, 246 (1990). 
[10] H. Davoudiasl, J. L. Hewett and T. G. Rizzo, Phys. Rev. Lett. 84, 2080 (2000) arXiv:hep-ph/9909255.

[11] C. Csáki, H. Ooguri, Y. Oz and J. Terning, JHEP 9901, 017 (1999) arXiv:hep-th/9806021.

[12] X. Amador and E. Cáceres, JHEP 0411, 022 (2004) arXiv:hep-th/0402061.

[13] H. Davoudiasl, J. L. Hewett and T. G. Rizzo, JHEP 0304, 001 (2003) arXiv:hep-ph/0211377.

[14] K. Shiraishi, Prog. Theor. Phys. 74, 832 (1985).

[15] S. Kachru, R. Kallosh, A. Linde and S. P. Trivedi, Phys. Rev. D 68, 046005 (2003) arXiv:hep-th/0301240.

[16] H. Firouzjahi and S. H. Tye, arXiv:hep-th/0512076. 